\title{
Lung and chest wall mechanics in ventilated patients with end stage idiopathic pulmonary fibrosis
}

\author{
Stefano Nava, Fiorenzo Rubini
}

\begin{abstract}
Background-Idiopathic pulmonary fibrosis is an inflammatory disease which leads to chronic ventilatory insufficiency and is characterised by a reduction in pulmonary static and dynamic volumes. It has been suggested that lung elastance may also be abnormally increased, particularly in end stage disease, but this has not been systematically tested. The aim of this study was to assess the respiratory mechanics during mechanical ventilation in patients affected by end stage disease. Methods-Respiratory mechanics were monitored in seven patients with idiopathic pulmonary fibrosis being ventilated for acute respiratory failure $\left(\mathrm{PaO}_{2} / \mathrm{Fio}_{2} 5.8\right.$ (0.3); pH 7.28 (0.02); $\mathrm{Paco}_{2} 8.44$ (0.82) kPa; tidal volume $3.4(0.2) \mathrm{ml} / \mathrm{kg}$; respiratory rate 35.1 (8.8) breaths/min) using an oesophageal balloon and airway occlusion during constant flow inflation. The total respiratory system mechanics (rs) was partitioned into lung (L) and chest wall (w) mechanics to measure static intrinsic positive end expiratory pressure (PEEPi), static (Est) and dynamic (Edyn) elastances, total respiratory resistance (Rrs), interrupter respiratory resistance (Rint,rs), and additional respiratory resistance ( $\Delta \mathrm{Rrs})$.
\end{abstract}

Results-PEEPi was negligible in all patients. Edyn,rs and Est,rs were markedly increased $(60.9(7.3)$ and $51.9(8.0) \mathrm{cm}$ $\mathrm{H}_{2} \mathrm{O} / 1$, respectively), and this was due to abnormal lung elastance (dynamic 53.9 (8.0) $\mathrm{cm} \mathrm{H} \mathrm{H}_{2} \mathrm{O} / 1$, static $46.1 \quad$ (8.1) cm $\left.\mathrm{H}_{2} \mathrm{O} / 1\right)$ while chest wall elastance was only slightly increased. Rrs and Rint,rs were also increased above the normal range (16.7 (4.5) and 13.7 (3.5) $\mathrm{cm} \mathrm{H}_{2} \mathrm{O} / 1 / \mathrm{s}$, respectively). RL and Rint,L contributed $88 \%$ and $89 \%$, on average, to the total. Edyn,rs, Est,rs, Rrs and Rint,rs were significantly correlated with the degree of hypercapnia $(r=0.64(\mathrm{p}<0.01), r=0.54$ $(\mathrm{p}<0.05), r=0.84(\mathrm{p}<0.001)$, and $r=0.72$ $(\mathrm{p}<0.001)$, respectively).

Conclusions-The elastances and resistances of the respiratory system are significantly altered in ventilated patients with end stage idiopathic pulmonary fibrosis. These features are almost totally due to abnormalities in lung mechanics. These profound alterations in elastic and resistive mechanical properties at this stage of the disease may be responsible for the onset of hypercapnia.

(Thorax 1999;54:390-395)

Keywords: idiopathic pulmonary fibrosis; mechanical ventilation; pulmonary mechanics

Idiopathic pulmonary fibrosis is a progressive and generally fatal disease. The essential histological feature is chronic inflammation of the alveolar wall which tends to destroy the lung architecture by consequent healing with progressively severe fibrosis. Alterations of static and dynamic lung volumes due to this disease are well known but little information exists about the mechanical characteristics. ${ }^{1}$ Studies on static and dynamic lung compliance performed in stable patients revealed that the lung is less distensible than normal, ${ }^{2-5}$ and it has been suggested that measurements of distensibility may correlate with degree of fibrosis ${ }^{2} 5$ even though studies performed in the late stage of the disease are lacking. As the disease progresses to the end stage, hypoxic respiratory failure ensues and mechanical ventilation is sometimes administered, especially in patients awaiting lung transplantation. ${ }^{6}$ The institution of mechanical ventilation allows us to record respiratory mechanics non-invasively. These have been shown to be useful both clinically to predict weaning from mechanical ventilation ${ }^{78}$ and physiologically to understand the mechanisms leading to life threatening episodes of acute respiratory failure. ${ }^{9-13}$ This study reports the first systematic measurement of resistances and elastances of the total respiratory system, lung, and chest wall during mechanical ventilation in patients with end stage idiopathic pulmonary fibrosis.

\section{Methods}

Seven mechanically ventilated patients with idiopathic pulmonary fibrosis, admitted to our Respiratory Intensive Care Unit for acute respiratory failure while on the waiting list for single lung transplantation, were studied. The study protocol was approved by the Salvatore Maugeri Institutional ethical committee and informed consent was obtained from the patients or from their next of kin.

\section{PATIENTS}

The clinical characteristics of the study population are shown in table 1. Pulmonary function tests presented in the table were the last performed in our department (1-5 months before the study). The clinical diagnosis of 
Table 1 Pulmonary function tests, arterial blood gas tensions and breathing pattern

\begin{tabular}{lll}
\hline Variables & & \% predicted \\
\hline Vital capacity (ml) & $810(123)$ & $19.2(8.8)$ \\
Forced expiratory volume in one second (ml) & $745(259)$ & $26.3(10.0)$ \\
Total lung capacity (TLC) (1) & $3.02(0.79)$ & $26.5(9.3)$ \\
Functional residual capacity (FRC) (1) & $1.26(0.93)$ & $37(12)$ \\
FRC/TLC & & $41.7(8.5)$ \\
$\mathrm{Carbon}$ monoxide transfer factor & $7.28(0.02)$ & $21.4(10.5)$ \\
pH & $5.8(0.3)$ & \\
$\mathrm{PaO}_{2} / \mathrm{FiO}_{2}$ & $8.44(0.82)$ & \\
$\mathrm{PaCO}_{2}(\mathrm{kPa})$ & $3.46(0.24)$ & \\
$\mathrm{Tidal}_{\text {volume }}(\mathrm{ml} / \mathrm{kg})$ & $35.1(8.8)$ & \\
Respiratory rate (breaths/min) &
\end{tabular}

The data are presented as mean (SD). All parameters were recorded just before intubation, except pulmonary function tests which were recorded before the episode of acute respiratory failure.

idiopathic pulmonary fibrosis was confirmed by transbronchial biopsy or after explorative thoracotomy, the diagnostic strategy used in most published studies. ${ }^{1}$ All the patients had undergone one of these two procedures to be included on the North Italian transplant waiting list. Computed tomographic (CT) scans showed interstitial reticular or linear opacities, patchy areas of ground glass appearance, and varying degrees of honeycomb changes. The patients were on long term oxygen therapy and were being treated with systemic corticosteroids or immunosuppressant drugs. Patients who had chest radiographic evidence of pneumonia, clinical or echocardiographic signs of heart failure, or pulmonary embolisms were excluded from the study. The decision to intubate was made by the attending physician on the basis of marked deterioration in blood gas tensions with hypercapnia $\left(\mathrm{pH} \leqslant 7.30\right.$ with $\left.\mathrm{PaCO}_{2} \geqslant 6.6 \mathrm{kPa}\right)$ and the presence of severe dyspnoea and tachypnoea. All the patients were intubated with an endotracheal tube $(7.0$ or $7.5 \mathrm{~mm}$ internal diameter) and were mechanically ventilated with a Cesar ventilator (Taema, France) using constant inspiratory flow. The study of respiratory mechanics was started within 24 hours of the intubation.

\section{MATERIALS}

The breathing pattern during spontaneous breathing, just before intubation, was measured using a portable Wright spirometer. Flow was measured with a heated pneumotachograph (Screenmate Jaeger, Wurzburg, Germany) inserted between the proximal end of the endotracheal tube and the "Y" of the ventilator. Volume was determined by numerical integration of the flow signal. Differential pressure transducers (Honeywell, Freeport, Illinois, USA, $\pm 250 \mathrm{~cm} \mathrm{H}_{2} \mathrm{O}$ ) were employed to record the pressures. Pressure at the airways (Paw) was sampled by means of tubing proximal to the pneumotachograph. Tracheal pressure (Ptr) was recorded with a polyethylene catheter with a few side holes at the distal tip, positioned in the trachea $3-4 \mathrm{~cm}$ below the distal end of the endotracheal tube. Oesophageal pressure (Poes) was monitored using a balloon catheter system inserted in the middle third of the oesophagus and filled with $0.8 \mathrm{ml}$ of air. The position of the balloon was checked using the so called "occlusion test" performed at the beginning of the study. ${ }^{14}$ The transpulmonary pressure (PL) was obtained by subtracting Poes from Ptr. The equipment dead
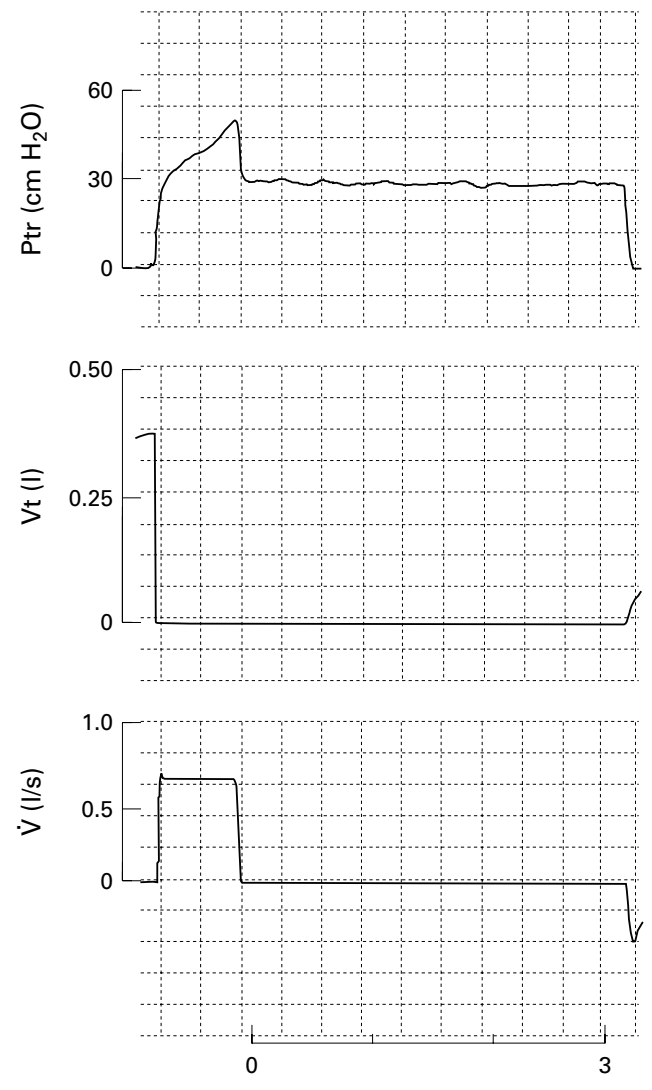

$\mathrm{t}(\mathrm{s})$

Figure 1 Typical record of a patient during the end inspiratory occlusion manoeuvre. Ptr = tracheal pressure, $V t=$ tidal volume, $\dot{V}=$ airflow. Note that an apparent plateau in Ptr was reached before three seconds.

space, not including the endotracheal tube, was $140 \mathrm{ml}$. Arterial blood gas tensions were measured with a blood gas analyser (Radiometer, Copenhagen, Denmark).

\section{STUDY PROTOCOL}

The patients were sedated with a benzodiazepine and curarised with pancuronium bromide $0.1 \mathrm{mg} / \mathrm{kg}$ during the study. They were examined in a semi-recumbent position. Airway suctioning was carefully done three minutes before each measurement. This time interval has been shown to be sufficient to avoid the effects of the transient bronchoconstriction response to suction. ${ }^{15}$ During the study a physician not involved in the procedure was always present to provide care for the patients. The ventilator settings consisted of a fixed tidal volume $(8.3(0.9) \mathrm{ml} / \mathrm{kg})$, a fixed inspiratory flow (0.60 $(0.2) \mathrm{l} / \mathrm{s})$, and a respiratory frequency of 12 breaths $/ \mathrm{min}$. The tidal volume was set to be between $30 \%$ and $40 \%$ of the total lung capacity (TLC) recorded in each patient during the previous pulmonary function test. This was likely to allow us to record the respiratory mechanics far away from the "flat" part of the pressure/volume curve ${ }^{3}$ and very close to that part of the curve in which these patients usually breathe spontaneously (table 1, FRC/TLC $41.7 \%)$. The inspired fraction of oxygen $\left(\mathrm{FiO}_{2}\right)$ was set to achieve an oxygen saturation $\left(\mathrm{SaO}_{2}\right)$ of $>90 \%$. All the patients were studied at zero end expiratory pressure (ZEEP). 


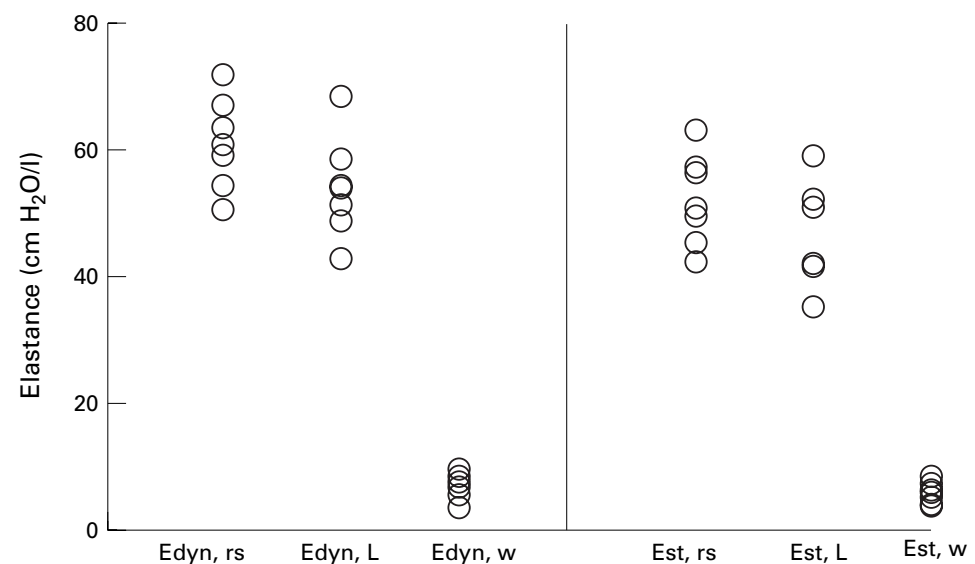

Figure 2 Individual values for dynamic and static elastances of the total respiratory system (Edyn,rs and Est,rs), lung (Edyn,L and Est,L) and chest wall (Edyn,w and Est,w).
(Edyn,w) was computed by subtracting Edyn,L from Edyn,rs.

We computed the flow resistive properties of the endotracheal tubes by dividing the difference between peak Paw and peak Ptr by the preceding constant inspiratory flow. ${ }^{9}$ The so called interrupter resistances of the total respiratory system (Rint,rs), lung (Rint,L), and chest wall (Rint,w) were computed by dividing (Pmax-P1) from the Ptr, PL, and Poes tracings, respectively, by the preceding constant flow and subtracting the resistance of the endotracheal tube. The slow decay in pressure (P1-P2) divided by the flow preceding the occlusion yields the effective additional resistance of the total respiratory system $(\Delta \mathrm{R}, \mathrm{rs})$, lung $(\Delta \mathrm{R}, \mathrm{L})$, and chest wall $(\Delta R, w)$. The sum of interrupter resistances and additional resistances represents the total resistance of the respiratory system (Rrs), lung ( $\mathrm{RL}_{\mathrm{L}}$ ), and chest wall (Rw). In the calculation of resistances the error due to the closing time of the valve of the ventilator was corrected. ${ }^{17}$

The end inspiratory ${ }^{16}$ and end expiratory occlusion $^{10}$ method was employed to record respiratory mechanics. Three to five measurements for each method were taken at fixed intervals of five minutes to assess the reproducibility of the measurements. Several regular mechanical breaths were recorded to achieve a steady state after which the respiratory mechanics were measured. Intrinsic end expiratory pressure (PEEPi) was measured by occluding the airway opening for five seconds at the end of tidal expiration and pressing the end expiratory hold button incorporated in the ventilator. During this occlusion the increases in Paw and Ptr, if any, were due to end expiratory elastic recoil of the respiratory system (PEEPi,rs) and the increase in PL reflected end expiratory elastic recoil of the lung $(\mathrm{PEEPi}, \mathrm{L})$. End expiratory elastic recoil of the chest wall (PEEPI,w) was computed by subtracting PEEPi,L from PEEPi,rs. After several breaths the airway opening was occluded at the end of inflation for three seconds. As shown in fig 1, three seconds were usually sufficient to reach an apparent plateau in tracheal pressure. Briefly, after the occlusion there is a sudden decrease in Paw, Ptr, and PL from a maximal value (Pmax) to a lower value (P1), followed by a gradual decrease until an apparent plateau is reached (P2). P1 was measured by back extrapolation of a computer fitted curve. P2 represents the static end inspiratory elastic recoil of the total respiratory system (Pst,rs), lung (Pst,L), and chest wall (Pst,w) on Paw, Ptr, PL, and Poes, respectively. The static elastance of the total respiratory system (Est,rs) was calculated as the ratio between the difference between P2 and end expiratory (PEEPi) plateau pressure on the Ptr trace and the expiratory tidal volume $(\mathrm{Vt})$. The static elastance of the lungs (Est,L) was recorded as the ratio described above, but using the PL trace. The static elastance of the chest wall (Est,w) was computed by subtracting Est,L from Est,rs. The dynamic elastances of the respiratory system (Edyn,rs), and lungs (Edyn,L) were measured by dividing P1-PEEPi on the Paw or Ptr and PL tracings, respectively, by Vt. The dynamic elastance of the chest wall

\section{DATA ANALYSIS}

The breathing pattern was analysed over a two minute period of spontaneous breathing in oxygen. All signals were recorded on an eight channel recorder (Gould TA 4000, Valley View, Ohio, USA). They were sampled at $100 \mathrm{~Hz}$ by an analogue-to-digital converter and stored on a personal computer (Vectra $386 / 20$ N, Hewlett Packard, USA) for subsequent analysis. The mean value from three measurements was used for each physiological variable. The results are expressed as mean (SD). Statistical analysis was performed using the Student's $t$ test for paired observations. Correlation analysis was used to assess the associations between variables. A p value of $<0.05$ was considered significant.

\section{Results}

Only one out of seven patients was extubated and passed to non-invasive ventilation five days after intubation, while the others died after 3.2 (4) days of ventilation. The causes of death were mainly related to hypoxia refractory to oxygen therapy. No signs of new pulmonary infections or increased lung water, compared to those on the initial radiograph, were observed before the patients died. One patient developed bilateral basal atelectasis 24 hours after the measurements of respiratory mechanics. Two of the patients also had multiple organ failure.

The breathing pattern immediately before intubation was characterised by an increased breathing frequency and reduced tidal volume (table 1). PEEPi was present in only one patient and was $1.3 \mathrm{~cm} \mathrm{H}_{2} \mathrm{O}$ for PEEPi,rs, $1.0 \mathrm{~cm} \mathrm{H}_{2} \mathrm{O}$ for PEEPi, L, and $0.3 \mathrm{~cm} \mathrm{H}_{2} \mathrm{O}$ for PEEPi,w.

Figure 2 shows the elastance of the respiratory system, lung, and chest wall during passive ventilation for each patient. Mean Edyn,rs was 60.91 (7.28) $\mathrm{cm} \mathrm{H}_{2} \mathrm{O} / 1$ while Edyn,L and Edyn,w were 53.90 (7.98) $\mathrm{cm} \mathrm{H}_{2} \mathrm{O} / 1$ and 7.01 (1.98) $\mathrm{cm} \mathrm{H}_{2} \mathrm{O} / 1$, respectively. Mean Est,rs was $51.91(7.22) \mathrm{cm} \mathrm{H}_{2} \mathrm{O}$ while the values for Est, $\mathrm{L}$ and Est,w were 46.08 (8.06) $\mathrm{cm} \mathrm{H}_{2} \mathrm{O} / 1$ 


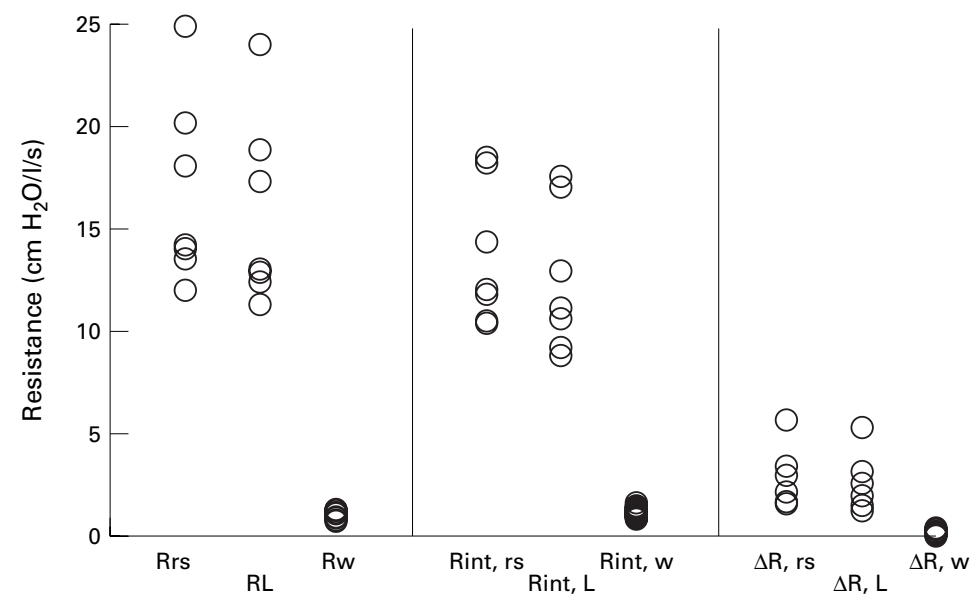

Figure 3 Individual values for the total resistances of the total respiratory system (Rrs), lung $\left(R_{L}\right)$ and chest wall (Rw), for the interrupter resistances of the total respiratory system (Rint,rs), lung (Rint, L) and chest wall (Rint,w), and for the effective additional resistance of the total respiratory system $(\Delta R, r s)$, lung $(\Delta R, L)$ and chest wall $(\Delta R, w)$.

and $6.02(0.80) \mathrm{cm} \mathrm{H}_{2} \mathrm{O} / 1$, respectively. The contribution of lung elastances to the respiratory system elastances amounted to $88 \%$ and $89 \%$ for Edyn,rs and Est,rs, respectively. Edyn,rs and Edyn,L were significantly higher than Est,rs and Est,L, respectively $(\mathrm{p}<0.05)$.

The resistances of the respiratory system, lung, and chest wall for each patient are shown
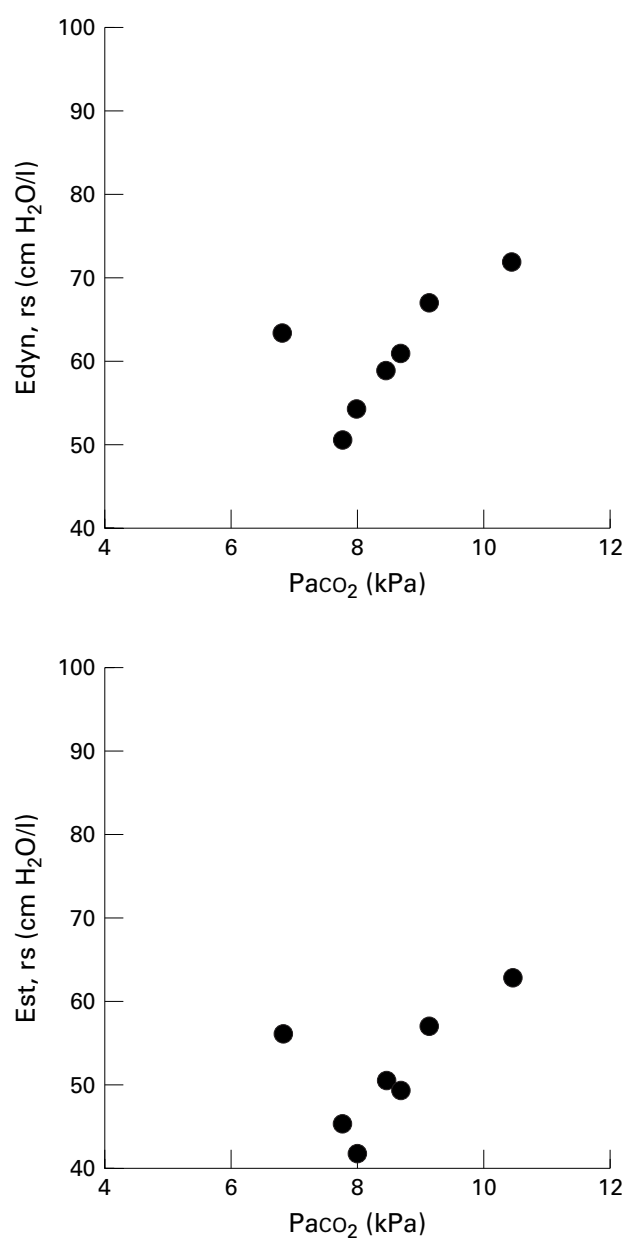

in fig 3. Mean Rrs was 16.70 (4.61) $\mathrm{cm} \mathrm{H}_{2} \mathrm{O} / 1 /$ $\mathrm{s}$, while $\mathrm{RL}_{\mathrm{L}}$ and $\mathrm{Rw}$ were 15.27 (4.61) and 1.14 (0.29) $\mathrm{cm} \mathrm{H}_{2} \mathrm{O} / \mathrm{l} / \mathrm{s}$, respectively. Rint,rs was 13.68 (3.45) $\mathrm{cm} \mathrm{H}_{2} \mathrm{O} / 1 / \mathrm{s}$ while the values for Rint,L and Rint,w were 12.47 (3.57) and 1.21 (0.24) $\mathrm{cm} \mathrm{H}_{2} \mathrm{O} / \mathrm{l} / \mathrm{s}$, respectively. $\Delta \mathrm{R}, \mathrm{rs}, \Delta \mathrm{R}, \mathrm{L}$ and $\Delta \mathrm{R}, \mathrm{w}$ were $3.02(1.42), 2.80$ (1.39), and $0.25(0.11) \mathrm{cm} \mathrm{H}_{2} \mathrm{O} / 1 / \mathrm{s}$, respectively.

Figure 4 shows the correlation analysis between the degree of alteration in mechanical variables and the level of $\mathrm{PaCO}_{2}$ recorded just before intubation. Edyn,rs Est,rs, Rrs, and Rint,rs were significantly correlated with the degree of hypercapnia $(r=0.64(\mathrm{p}<0.01), 0.54$ $(\mathrm{p}<0.05), 0.84(\mathrm{p}<0.001)$ and $0.72(\mathrm{p}<0.001)$, respectively). Tidal volume (Vt) and breathing frequency ( $f$ ) were also significantly correlated with some mechanical variables, but these correlations were rather weak (Vt and Rint,rs, $r=$ $0.38(\mathrm{p}<0.05) ; \mathrm{Vt}$ and Est,L,rs, $r=0.41$ $(\mathrm{p}<0.05)$; $\mathrm{f}$ and Rrs, $r=0.39(\mathrm{p}<0.05)$; $\mathrm{f}$ and Edyn,rs, $r=0.46(\mathrm{p}<0.05))$.

\section{Discussion}

In this study we have provided information for the first time about partitioning of elastic and resistive properties of the total respiratory system between lung and chest wall mechanics in
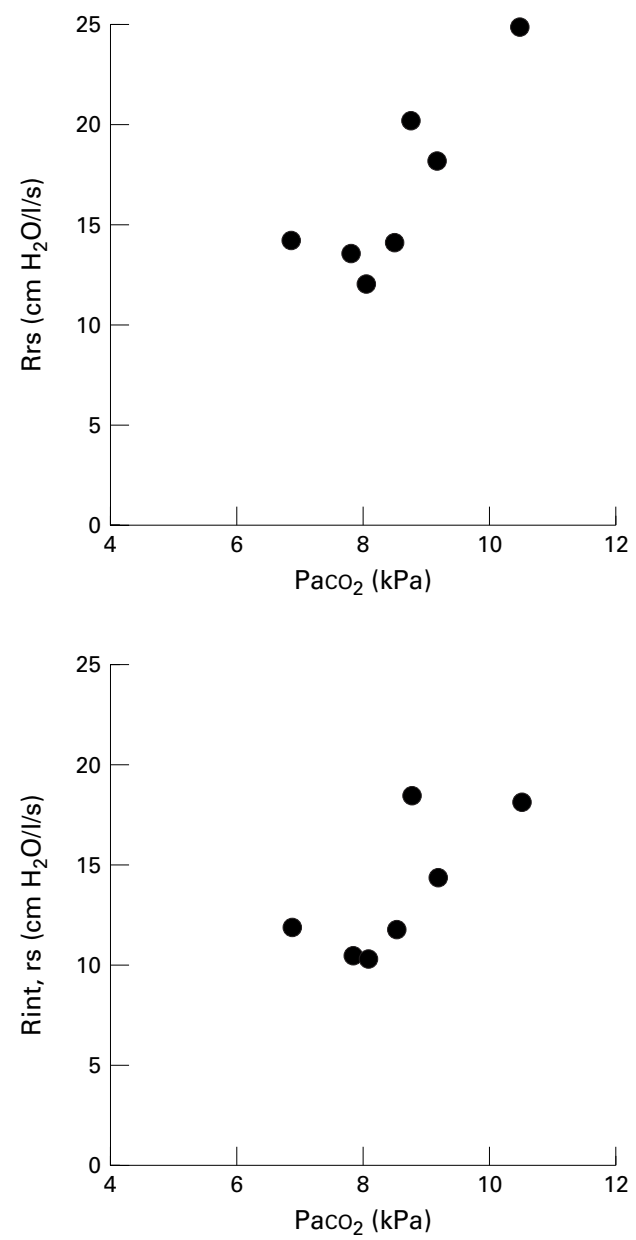

Figure 4 Correlation analysis between dynamic (Edyn,rs) and static (Est,rs) elastances of the respiratory system, total resistances (Rrs) and interrupter resistances (Rint,rs) of the total respiratory system, and arterial carbon dioxide tension $\left(\mathrm{PaCO}_{2}\right)$. 
mechanically ventilated patients with end stage idiopathic pulmonary fibrosis.

Idiopathic pulmonary fibrosis is a progressive disease. The median survival from the time symptoms are first reported is less than five years, irrespective of the response to treatment. ${ }^{18}{ }^{19}$ Late stages of the disease are characterised by breathlessness, hypoxia, and hyperventilation. When acute respiratory failure ensues, most of the patients develop hypercapnia so that death occurs unless mechanical ventilation is instituted. Mechanical ventilation offers us the unique possibility of measuring passive respiratory mechanics non-invasively while the patients are ventilated. Information is available in the literature concerning most disease of the respiratory system such as chronic obstructive pulmonary disease (COPD), ${ }^{8-13}$ adult respiratory distress syndrome (ARDS), ${ }^{71}$ postoperative complications,${ }^{11}$ and cardiogenic pulmonary oedema, ${ }^{10}{ }^{13}$ but there are no data recorded from patients with interstitial lung disease. We have found that this last group of patients has gross abnormalities of pulmonary mechanics.

A significant increase in the elastance of the respiratory system was observed. The mean value reported in the present study is approximately four times that recorded in normal anaesthetised subjects ${ }^{20}$ and even higher than in a group of patients with ARDS who did not survive an episode of acute respiratory failure. ${ }^{7}$ The increase in elastance of the respiratory system was mainly due to the abnormally increased lung elastance. Some studies performed in spontaneously breathing subjects have already reported that dynamic or static compliance of the lung (the reciprocal of elastance) is reduced. ${ }^{25}$ These studies were performed in spontaneously breathing, apparently stable, patients. For example, the largest study reported was in 23 subjects $^{2}$ with mild resting hypoxaemia, more than one third of whom had normal values of $\mathrm{PaO}_{2}$. Nevertheless, the authors of the study reported that a reduction in lung distensibility was significantly correlated with the severity of the morphological stage of the disease. There are many reasons why lung elastance is so markedly increased. Interstitial pulmonary fibrosis is characterised by severe alveolar fibrosis which reduces lung volumes. With the simple loss of lung volume the pressure/volume curve of the lung is contracted on its volume axis, therefore reducing the static or dynamic compliances. However, part of the explanation of the increased elastance may also be related to a "true" stiffness of the ventilated lung. Problems of volume correction may be avoided by fitting an exponential expression to the curve to obtain a shape factor (k), but this does not clarify the issue. ${ }^{5}$ It has also been suggested that loss of compliance may be due to the decreased amount of surfactant phospholipid present in patients with interstitial pulmonary fibrosis. ${ }^{21}$ Chest wall elastance only accounted for about $15 \%$ of the increase in the elastance of the respiratory system but it was, nevertheless, slightly above the normal value, probably due to the increased lung recoil which may displace the chest wall into the lower flat portion of the pressure/volume curve.

The resistance of the respiratory system was also markedly increased compared with the values reported for normal subjects, ${ }^{20}{ }^{22}$ although to a lesser extent than that reported for patients with COPD. ${ }^{8-13}$ Lung resistances accounted for most of the total amount. It is rather surprising that Rint, estimation of airway resistance, was so high in patients with increased lung recoil, which should theoretically increase airway calibre. Interestingly, West and Alexander ${ }^{23}$ found that patients with higher respiratory work per breath also had increased resistive work compared with normal subjects. Indeed, Gibson and Pride ${ }^{3}$ suggested that the decreased lung volume observed in their patients with fibrosing alveolitis could result in a decrease in upstream conductance, even without structural changes in the airway which are, however, likely to be present in our patients affected by end stage disease. An interesting finding of our study is the increase in $\Delta \mathrm{R}$ that reflects the viscoelastic properties and the time-constant heterogeneity within the respiratory tissue. Idiopathic pulmonary fibrosis in its late stages is characterised by patchy pathological findings so that advanced fibrotic changes occur in some areas, especially subpleurally, whereas other areas have more activity. ${ }^{24}$ These different histological features within the same interstitial tissue may explain this increase in $\Delta \mathrm{R}$ since it is likely that, together with functionally deranged alveoli, some others may at least be partially preserved which may lead to time-constant heterogeneities.

Some methodological problems should be discussed. Firstly, the values of the mechanics of the respiratory system obtained during mechanical ventilation may not truly represent the values that occur during spontaneous breathing. This is particularly true for the measurements of chest wall mechanics which are likely to be affected by curarisation and sedation. Westbrook et $a l^{25}$ found that the static volume/pressure curve of the chest wall either shifts to the right or becomes less curved at low lung volumes with induction of anaesthesia paralysis. The measurements of respiratory mechanics must, however, be performed with the patients sedated to avoid any active contribution which would profoundly alter the recordings. Curarisation has been shown not to affect elastance or resistance of the respiratory system further after anaesthesia. ${ }^{26}$ Secondly, the end expiratory occlusion was held in this study for three seconds. This time interval has been used in most of the studies in the literature $^{91027}$ although recent recommendations are for a five second interval to allow true static recoil conditions to be reached. ${ }^{28}$ As shown in fig 1 , three seconds were usually enough to reach an apparent plateau in tracheal pressure, even though occlusion had to be prolonged in one patient who reached this plateau later. While three seconds may not be enough in most patients with COPD due to important time-constant heterogeneity, it should be sufficient for patients with idiopathic pulmonary fibrosis in whom we have shown the 
change in resistance which reflects this heterogeneity to be about $30 \%$ lower than in patients with COPD. ${ }^{9}$ Thirdly, we examined only one setting of the ventilator. It has been shown in normal anaesthetised paralysed subjects that Rint,rs increases linearly with increasing flow and so the fixed inspiratory flow used in the present study (0.60 (0.2) 1/s) may be higher than that produced during spontaneous breathing. While this is true for normal subjects, it is not necessarily the case in patients with increased respiratory resistance in whom Eissa and coworkers ${ }^{27}$ found that resistance and static elastance did not change with increasing inspiratory flow.

The results of this study confirm the abnormal respiratory mechanics in patients with idiopathic pulmonary fibrosis. Most of the abnormalities are likely to be a reflection of the progressive inflammatory process that leads to interstitial fibrosis and reorganisation of the lung architecture. Since alveolar hypoventilation is uncommon in these patients before death, it is possible to speculate that, during the course of the disease, inspiratory muscles have the time required to adjust mechanically and metabolically to the increased workload. In the very end stages of the disease the lungs are likely to become so stiff and the resistances increase to such a point that the respiratory muscles can no longer sustain the loads. The breathing pattern would therefore be characterised by the typical rapid shallow breathing pattern present in all our patients before intubation, leading to progressive hypercapnic respiratory failure. Some of the correlations found between the degree of hypercapnia and respiratory mechanics, even though rather weak, may indirectly suggest that the more severe is the alveolar hypoventilation, the greater the degree of mechanical changes.

1 Katzenstein ALA, Myers J. Idiopathic pulmonary fibrosis Clinical relevance of pathologic classification. Am $\mathcal{F}$ Respir Crit Care Med 1998;157:1301-15.

2 Fulmer JD, Roberts WC, von Gall ER, et al. Morphologicphysiologic correlates of the severity of fibrosis and degree of cellularity in idiopathic pulmonary fibrosis. 7 Clin Invest 1979;63:665-76.

3 Gibson GJ, Pride NB. Pulmonary mechanics in fibrosing alveolitis. Am Rev Respir Dis 1977;116:637-47.

4 Murphy DMF, Hall DR, Petersen MR, et al. The effect of diffuse pulmonary fibrosis on lung mechanics. Bull Eur diffuse pulmonary fibrosis on lun
Physiopathol Respir 1981;17:27-41.

5 Sansores RH, Ramirez-Venegas A, Perez-Padilla R, et al. Correlation between pulmonary fibrosis and the lung pressure-volume curve. Lung 1996;174:15-23.
6 Trulock EP. Lung transplantation. Am 7 Respir Crit Care Med 1997; 155:789-818.

7 Mancebo J, Benito S, Martin M, et al. Value of static pulmonary compliance in predicting mortality in patients with acute respiratory failure. Intensive Care Med 1988;14:1104.

8 Zanotti E, Rubini F, Iotti G, et al. Elevated static compliance of the total respiratory system: early predictor of weaning unsuccess in severe COPD patients mechanically ventilated. Intensive Care Med 1995;21:399-405.

9 Jubran A, Tobin MJ. Passive mechanics of lung and chest wall in patients who failed or succeeded in trials of weaning. Am f Respir Crit Care Med 1997;155:916-21.

10 Polese G, Rossi A, Appendini L, et al. Partitioning of respiratory mechanics in mechanically ventilated patients. $\mathcal{F}$ Appl Physiol 1991;71:2425-33.

11 Nava S, Bruschi C, Fracchia C, et al. Patient-ventilator interaction and inspiratory effort during pressure support ventilation in patients with different pathologies. Eur Respir f 1997; 10:177-83.

12 Nava S, Bruschi C, Rubini F, et al. Respiratory response and inspiratory effort during pressure support ventilation in COPD patients. Intensive Care Med 1995;21:871-9.

13 Broseghini C, Brandolese R, Poggi R, et al. Respiratory mechanics during the first day of mechanical ventilation in patients with pulmonary edema and chronic airway obstruction. Am Rev Respir Dis 1988;138:355-61.

14 Baydur A, Behrakis PK, Zin WA, et al. A simple method for assessing the validity of the esophageal balloon technique. Am Rev Respir Dis 1982;126:788-91.

15 Guglielminotti J, Desmonts JM, Dureuil B. Effects of tracheal suctioning on respiratory resistances in mechanically ventilated patients. Chest $1988 ; 113: 1335-8$.

16 Bates JHT, Rossi A, Milic-Emili J. Analysis of the behaviour of the respiratory system with constant inspiratory flow. $\mathcal{F}$ Appl Physiol 1985;64:441-50.

17 Kochi T, Okubo S, Zin WA, et al. Flow and volume dependence of pulmonary mechanics in anesthetized cats. $\mathcal{F} A p p l$ Physiol 1986;64:441-50.

18 Stack BHR, Choo-Kang YFJ, Heard BE. The prognosis of cryptogenic fibrosing alveolitis. Thorax 1972;27:535-42.

19 Rudd RM, Haslam PL, Turner-Warwick M. Cryptogenic fibrosing alveolitis. Relationship of pulmonary physiology and BAL to response to treatment and prognosis. Am Rev Respir Dis 1981;124:1-8.

20 D'Angelo E, Robatto F, Calderini E, et al. Pulmonary and chest wall mechanics in anesthetized paralyzed humans. $f$ Appl Physiol 1991;70:2602-10.

21 Robinson PC, Watters LC, King TE, et al. Idiopathic pulmonary fibrosis. Abnormalities in bronchioalveolar lavage fluid phospholipids. Am Rev Respir Dis 1988;137:58591 .

22 D'Angelo E, Calderini E, Torri G, et al. Respiratory mechanics in anesthetized paralyzed humans: effects of flow, volume, and time. F Appl Physiol 1989;67:2556-64.

23 West JR, Alexander JK. Studies on respiratory mechanics and the work of breathing in pulmonary fibrosis. $\mathrm{Am} \mathcal{F} \mathrm{Med}$ 1959;5:529-44.

24 Wintherbauer RH, Hammar SP, Hallman KO, et al. Diffuse interstitial pneumonitis. Clinicopathologic correlations in 20 patients treated with prednisone/azathioprine. Am $\mathcal{f}$ Med 1978;65:661-72.

25 Westerbrook PR, Stubbs SE, Sessler AD, et al. Effects of anesthesia and muscle paralysis on respiratory mechanics in normal man. F Appl Physiol 1973;34:81-6.

26 Behrakis PK, Higgs BD, Baydur A, et al. Respiratory mechanics during halothane anesthesia and anesthesiaparalysis in humans. F Appl Physiol 1983;55:1085-92.

27 Eissa NT, Ranieri VM, Corbeil C, et al. Analysis of behavior of the respiratory system in ARDS patients: effects of flow, volume and time. $\mathscr{f}$ Appl Physiol 1991;70:1719-29.

28 D'Angelo E, Calderini E, Robatto FM, et al. Lung and chest wall mechanics in patients with acquired immunodefiwall mechanics in patients with acquired immunodefi-
ciency syndrome and severe Pneumocystis carinii pneumonia. Eur Respir f 1997;10:2343-50. 